Soc. hoy 27: 44-56, 1er Sem. 2020 ISSN 0719 - 9554

\title{
Caras y contracaras de la institucionalización de la diversidad sexual en América Latina
}

Sides and counter-sides of the institutionalization of sexual diversity in Latin America

\section{SEBÁSTIAN GIRALDO-AGUIRRE* \& MATEO PAZOS CÁRDENAS ${ }^{* *}$}

Resumen: El presente artículo plantea una reflexión acerca de las repercusiones, tanto políticas como académicas, de la introducción de una agenda institucional que pretende representar las apuestas reivindicativas de la diversidad sexual. Para este objetivo, en un primer apartado se presenta una síntesis sobre la aparición del tema en el calendario legislativo de los Estados, posteriormente se analiza la lógica implícita en la construcción de los sujetos políticos a los cuales están dirigidos estas medidas, más adelante se detallan algunos efectos adversos que han causado estas estrategias. Finalmente, se presentan las reflexiones suscitadas a partir de los temas que se abordaron a lo largo del texto.

Palabras clave: Diversidad sexual, institucionalización, políticas públicas, LGBT.

\begin{abstract}
This paper proposes a reflection on the implications, both political and academic (epistemological and methodological), of the introduction of a corporate agenda that seeks to represent the claiming stakes of sexual diversity. For this purpose, in the first section, a summary of the topic's appearance in the legislative calendar of the States will be presented, then the implicit logic in the construction of the political subjects to whom these measures are aimed will be discussed, later some adverse effects that have caused these strategies will be detailed. Finally, some reflections arising from the issues discussed throughout the text will be presented.
\end{abstract}

\footnotetext{
* Sebastián Giraldo-Aguirre: Docente Universidade Estadual de Campinas, São Paulo, Brasil. Código ORCID: org/0000-0003-4207-2806 Contacto: s.giraldoaguirre@gmail.com

** Mateo Pazos Cárdenas: Docente Universidade Estadual de Campinas, São Paulo, Brasil. Código ORCID: org/0000-0002-4932-6736 Contacto: mpazoscardenas@gmail.com
} 
Key words: Sexual diversity, institutionalization, public policies, LGBT.

\section{Introducción}

T a institucionalización de la diversidad sexual hace referencia al paquete de medidas _. promoción y defensa de los derechos humanos de las identidades y sexualidades no heteronormativas. El panorama actual en América Latina es disímil: algunos países presentan avances legislativos y jurídicos significativos en lo referente a los derechos civiles de seguridad social para parejas homosexuales - pensión, afiliación al sistema de salud-, la posibilidad de contraer matrimonio y la adopción de menores de edad (Brasil, México, Uruguay, Argentina, Colombia), mientras que otros se encuentran más rezagados (Paraguay, Perú, Bolivia) y algunos incluso cuentan con leyes que condenan las prácticas homosexuales con varios años de cárcel (un buen número de países del Caribe como Jamaica, Belice y Trinidad y Tobago). A pesar de estas diferencias, la región actualmente está inmersa en un proceso político que a la vez que está siendo promovido y defendido desde diversos sectores y movimientos sociales (no necesariamente adscritos o calificados bajo las siglas "LGBT"), debe ser reflexionado a la luz de un análisis crítico que dé cuenta de la compleja trama de usos, significados, subjetividades y estrategias socioculturales y políticas que se entretejen alrededor del diálogo entre los Estadosnación y su ciudadanía.

La discusión sobre la diversidad sexual conlleva un análisis crítico de las implicaciones epistemológicas y políticas que trae consigo la entrada de este debate en el terreno de las decisiones políticas de los Estados-nación latinoamericanos. El fomento de políticas públicas puede constituirse en un arma de doble filo: por una parte, puede promover la defensa universal de los derechos, pero por otro, puede acaparar el proceso reivindicativo bajo una lógica institucional que deja de lado una serie de subjetividades y expresiones intrínsecas de la diversidad sexual misma que parecen reducirse y englobarse dentro una etiqueta nominal simplificadora/normalizadora: "lo LGBT" o, aún más, "la comunidad LGBT".

La incorporación de la diversidad sexual al terreno gubernamental se remonta a los años ochenta del siglo XX, debido principalmente a la epidemia del VIH/SIDA. Aunque las redadas y movilizaciones ocurridas a finales de los sesenta en Estados Unidos y Europa se habían constituido en un referente fundamental para la aparición de movimientos sociales homosexuales, fue hasta la proliferación de esta enfermedad que los Estados les prestan una atención especial a estos sectores sociales. El origen de la preocupación, entonces, no obedeció a un interés por remediar las demandas que algunos grupos venían reclamando, sino por un asunto de salud pública. 
En América Latina, particularmente, este fue un periodo de declive de los movimientos sociales que apenas estaban floreciendo (Díez, 2010); los que no desaparecieron, entraron en diálogo con las instancias gubernamentales para hacer frente a la epidemia (Vieira y Pires, 2014), dejando de lado el paquete de mociones y demandas que venían concertando. Posteriormente, esta coyuntura coincide con un proceso de democratización impulsado por la universalización de los derechos humanos después de la caída del muro de Berlín (Núñez Noriega, 2011) y con un periodo en el que se consolida con mayor fuerza el discurso sobre los derechos sexuales y reproductivos.

Por estas razones, la década de los noventa se constituye en el periodo propicio para el establecimiento de las bases políticas para la promoción de la política "LGBT", entre ellas, la promulgación de reformas constitucionales en la región (como es el caso de Colombia -1991-, México -1992-, Ecuador -1998-, por mencionar algunas), que buscaban incluir diversos sectores de sus poblaciones dentro del espacio político nacional que anteriormente habían estado olvidados por cuenta de las anteriores constituciones políticas decimonónicas. No obstante, fue hasta la década siguiente que se consolida la agenda política sobre la diversidad sexual a nivel mundial, aunque con algunos retrasos a escala regional, gracias a esfuerzos como los principios de Yogyakarta que "surgen de la necesidad de coherencia de las máximas de no discriminación consagradas en los propósitos de la Organización de las Naciones Unidas, los cuales hablan de raza, sexo, idioma o religión, mas no de preferencia o identidad sexual" (Estefan, 2013, p. 185).

En términos metodológicos, para plantear la reflexión del presente artículo se realizó una revisión de las iniciativas políticas que cobijan a las poblaciones con identidades o sexualidades diversas en diferentes países de América Latina, principalmente, en México, Colombia y Chile; además, se construyó un balance sobre la producción académica acerca del tema. Los análisis planteados a lo largo del documento también son resultado de los dilemas que han afrontado los autores en sus experiencias de investigación concentradas en los estudios de género y étnico/raciales.

\section{¿Qué sujeto se encuentra implícito bajo el proceso de institucionalización de la diversidad sexual?}

Diversos estudios feministas de finales del siglo $\mathrm{XX}$ se preocuparon por analizar las políticas públicas promovidas por procesos como el MED, GED e IPEG ${ }^{1}$ (MacKinnon,

\footnotetext{
${ }^{1}$ Mujeres en el Desarrollo -MED- fue una estrategia impulsada en la década de 1970 para incorporar a las mujeres dentro de los programas de desarrollo económico y así equilibrar sus derechos respecto a los hombres. En la década de los ochenta, surge el GED -Género en el Desarrollo- como una plataforma que involucra al carácter relacional del género en los programas de desarrollo. Por último, la Institucionalización de la Perspectiva de Género -IPEG- se constituye en una estrategia que intenta impactar en el aparato público y en la cultura institucional de las organizaciones, promoviendo la formulación de políticas públicas o directrices que propendan por la equidad de género.
} 
1995; Pateman, 1996; Phillips, 1996; Mouffe, 1999), indagando sus alcances y sus limitaciones, señalando el carácter liberal de estas políticas y subrayando, a partir de una perspectiva interseccional, sus incapacidades para convertirse en una reforma profunda para la situación de las mujeres, en especial, en los países en desarrollo. Precisamente, una de las premisas de las que partieron los análisis feministas fue cuestionar la presunción de una ciudadanía universal y el modelo hegemónico de "Mujer Universal" (Viveros Vigoya, 2009), argumentos que dejaban de lado las diferencias producidas por asuntos de género, etnia, clase social, edad o nacionalidad, situación que, según Phillips (1996), limitaba el reconocimiento de la diferencia e impedía el otorgamiento de poder a los grupos desfavorecidos.

Teniendo en cuenta estos antecedentes, es pertinente realizar ese mismo ejercicio con las iniciativas dirigidas a la diversidad sexual. ¿Qué sujeto se encuentra implícito en el proceso de institucionalización de la diversidad sexual? Las políticas sobre diversidad sexual son lineamientos que también regularizan y presumen la existencia de una persona LGBT "universal" (Dubel y Hielkema, 2010), construida bajo el marco jurídico y normativo del Estado moderno ${ }^{2}$. La integración arbitraria de asuntos sobre identidades de género -o sexuales- y orientaciones sexuales en un mismo "paquete", sin distinguir las diferencias que puede acarrear su tratamiento, es un error común de estas iniciativas, situación que denota el carácter excluyente de la sigla LGBT y la endodiscriminación que produce la misma.

La sigla LGBT se ha constituido en la bandera de este proceso de institucionalización. Sin embargo, más allá de la crítica señalada anteriormente, hay que impugnar cada una de las letras contenidas en ella y las nominaciones arbitrarias que establecen. Las categorías de lesbiana, gay, bisexual y población trans son denominaciones producto de reflexiones académicas provenientes del hemisferio norte y propias de contextos urbanos que, tal vez, no se corresponden con las identidades y sexualidades no normativas de otros contextos, como el latinoamericano. ¿Cómo nombrar las sexualidades diversas que ocurren -muy silenciosamente, por demás- entre las comunidades indígenas, afros o campesinas de nuestros países? ¿Son efectivamente "gays" o "bisexuales"? ¿Estos términos significan algo para aquellas personas? ¿Hacen parte de la "comunidad "LGBT"? Incluso una categoría como la queer ("cuir"), que irrumpe como una noción más abierta para nombrar y pensar las sexualidades y las identidades, debe ponerse en duda, pues puede convertirse en un vocablo improcedente, en términos de que responde más a ciertos intereses políticos y epistemológicos relacionados con discusiones académicas, que a las realidades que vive el grueso de las personas que, de una u otra manera, caen dentro de la significación y uso político de esta denominación.

\footnotetext{
2 Entendiendo el Estado moderno como "el pacto entre los varones para el ejercicio del poder, poder que se expande y se establece a través de instituciones que dan forma al patriarcado, que, con la acción articulada de sus tecnologías, generan verdades y se ejerce control sobre los cuerpos, las relaciones y la sexualidad (Pateman, 1995, p. 11).
} 
Igualmente, es importante cuestionar el concepto "comunidad LGBT", comúnmente usado para referirse "grupalmente" a las personas con sexualidades e identidades diversas, que parece erróneo si retomamos el análisis propuesto por Benedict Anderson (1993) en su texto "Comunidades imaginadas", en donde define las comunidades como agrupaciones de individuos que se aceptan como iguales ("horizontal” y "fraternalmente") así no se conozcan entre sí en su totalidad. Aunque Anderson plantea esta definición en su estudio sobre la constitución de naciones, podemos hacer uso de esta conceptualización para dar cuenta del equívoco en nominar a las personas con sexualidades diversas como una "comunidad", en la medida que no se tienen en cuenta los procesos de endodiscriminación entre estas personas (tanto los más visibles -como la discriminación hacia personas trans- como los menos evidentes: entre varones que se identifican como "gais", pero que se diferencian en términos de clase, etnia o expresión de género -ser afeminado, "loca", "joto", "fleto"-, entre otros), indicio que denota la imposibilidad de reconocerse como iguales, así como tampoco son reconocidas como tal por el resto de la ciudadanía.

Partiendo de lo anterior, es necesario añadir que en el proceso de construcción de los sujetos sexualmente diversos como "objetos de deseo para el Estado" (Gil Hernández, 2013) se ha desestimado la diversidad étnico-cultural de los países de la región y los consecuentes procesos de racialización de la sexualidad y sexualización de la raza (Nagel, 2000; Hill Collins, 2004; Gil Hernández, 2008; Viveros Vigoya, 2009; Wade 2013), siendo estos elementos fundamentales para esta discusión ${ }^{3}$. En el contexto latinoamericano, la noción de mestizaje se ha constituido como el mito fundacional de la gran mayoría de las poblaciones de la región, articulando y configurando mutuamente un control racial y socio-sexual para naturalizar ciertas diferencias y ubicarlas en un lugar social de exclusión e inferiorización (Viveros, 2009; Wade, 2013). Es por esto que la perspectiva interseccional enfatiza en la necesidad de dar cuenta de las diversas maneras en que operan las estructuras de dominación y las relaciones de poder entre sujetos(as) y entre las instituciones sociales, evidenciando la articulación de los sistemas sexo/género y raza/etnia como operantes dentro de un mismo repertorio de políticas y estereotipos que legitiman las desigualdades sociales de los sujetos(as) asumidos(as) como "diferentes" (Nagel, 2000; Hill Collins, 2004; Viveros Vigoya, 2009). Así como la heteronormatividad asume la estigmatización de las prácticas sexuales diversas para fundamentar su legitimidad, el orden racial hegemónico mestizo requiere la estigmatización de las prácticas sexuales de sus alteridades (indígenas o afrodescendientes) para reproducir su lugar de poder.

Asimismo, a pesar de que los proyectos impulsados por las organizaciones y las políticas aprobadas por varios Estados intentan impactar la población en general, desconocen los niveles de apropiación y significación de los mismos por parte de los

\footnotetext{
3 Para un análisis detallado de las diversas maneras en que se imbrican las categorías de sexo, género, identidad sexual, etnia, raza y clase en diferentes países, ver el compilado realizado por Wade, Urrea Giraldo y Viveros Vigoya (2008).
} 
diferentes sectores socioeconómicos: a grandes rasgos, parece que estos avances han repercutido con más eficacia en sectores mestizos de clases medias-altas y con niveles educativos altos mientras que la población restante ha quedado relegada. Aunque es un tema que puede tener varias interpretaciones, se debe hacer énfasis en la lógica implícita en el proceso de institucionalización. Las concesiones y términos contenidos en las agendas no atañen los marcos de sentido de los diferentes sectores sociales, sino que intenta ponderarlos regularmente bajo una perspectiva de clase y étnico-racial.

Otro tema señalado por los estudios sobre la IPEG (Maceira y Rayas, 2007) es la repercusión de los organismos internacionales en la promoción de cierto tipo de políticas en América Latina, entre las que se encuentran las iniciativas de género. Los procesos de globalización iniciados en la última mitad del siglo XX han hecho que los países del llamado "tercer mundo" adapten sus agendas e instituciones a la nueva política económica globalizada promulgada por instituciones hegemónicas como el Fondo Monetario Internacional, el Banco Mundial o la Organización de Naciones Unidas. Este tipo de organismos multilaterales establecen unas prioridades y unas políticas (de corte progresista y a la vez neoliberal) que no necesariamente se concatenan con las realidades sociales y políticas de los Estados de la región. Sus propuestas, por lo general, no tienen en cuenta la particularidad histórica y cultural de los países, tanto así que podrían catalogarse como proyectos con indicios neocolonizadores, dando pie al "imperialismo de la democracia sexual", entendido, en términos de Fassin (2009), como un proceso de apropiación de la libertad y de la igualdad del género y la sexualidad como emblemas de la modernidad democrática. De esta manera, la puesta en marcha de la institucionalización de la diversidad sexual en la región se debe considerar también como una estrategia que busca un beneplácito de las miradas internacionales para así ganar réditos y financiamientos por parte de estos organismos.

Por otra parte, la implementación de plataformas legislativas y jurídicas en defensa de la diversidad sexual está condicionada por la voluntad política de los gobiernos locales o nacionales de turno y no obedece a una "política de Estado". En el caso mexicano, por ejemplo, los avances legislativos se han logrado gracias a gobiernos encabezados por un sólo partido y en algunos estados de la República (debido a la condición federal de la misma), por lo que la implementación no es de alcance nacional y no es producto de una voluntad general del Estado. En otros casos, como Colombia, el proceso no obedece a decisiones legislativas (generalmente entorpecidas por argumentos soportados en posiciones morales y religiosas), sino a litigios estratégicos que, por medio de fallos jurídicos impugnados por la ciudadanía y resueltos por altas instancias (como la Corte Constitucional), han logrado algunas reivindicaciones como el matrimonio y la adopción.

A lo largo de este apartado, hemos tratado de poner sobre la mesa diferentes aspectos que deben ser analizados cuidadosamente a la hora de pensar los términos a través de los cuales se instala la discusión sobre las diversidades sexuales en los Estadosnación latinoamericanos en la actualidad y de cómo se constituyen los sujetos que son 
objeto de estas discusiones en términos legislativos y políticos. A continuación, propondremos algunas consecuencias adversas que emergen en la promulgación de estas políticas.

\section{Desenlaces adversos en el proceso de institucionalización de la "ciudadanía LGBT"}

El proceso de institucionalización suscita un primer cuestionamiento: ¿dónde quedó el proceso libertario y transformador de los movimientos homosexuales? Parece que a medida que se consolidan políticamente los "asuntos LGBT", al mismo tiempo se "normaliza" la diversidad sexual como condición de acceso de estos sujetos a la plena ciudadanía (Gil Hernández, 2013), es decir, se le arrebata la potencia transformadora que aparentemente contenía en su interior. La emergencia de los movimientos sociales en defensa de las sexualidades e identidades no normativas nacieron con un espíritu revolucionario que pretendía alterar el orden sexual arraigado culturalmente; sus planteamientos y acciones estaban dirigidas a desestabilizar las subordinaciones provocadas por la heterosexualidad normativa y la dominación masculina. En la actualidad, como lo subrayan varios autores (Aparicio Erazo, 2009; De la Dehesa, 2010: Gil Hernández, 2013, Bustamante Tejada, 2013), el activismo aparece constreñido y crecientemente racionalizado por la lógica tecnocrática, que es funcional al régimen biopolítico heteronormativo y neoliberal.

Con el paso del tiempo, el espíritu rebelde de los movimientos comienza a desdibujarse y entra en un periodo de negociación con las instancias políticas. Se debe tener en cuenta que esta etapa obedece a un modelo de desarrollo neoliberal que trae consigo un proceso de despolitización que empobrece las dinámicas reivindicativas de los movimientos sociales. En particular, la agenda promovida por los grupos homosexuales y trans se depura, respondiendo a una lógica profiláctica de los Estados y limpiándose de cualquier indicio de desorden moral. Asuntos como la salud sexual, la monogamia y los modelos de familia, se constituyen en los soportes bajo las cuales el orden político integra cualquier demanda de género, entre ellas, las prescritas por la diversidad sexual. Tiene lógica, así, que las exigencias que más resuenen mediáticamente sean las relacionadas con el matrimonio y la adopción; otros contenidos como las condiciones laborales, la violencia física y simbólica mediada por la homofobia y la transfobia, el ingreso a instituciones educativas y la apropiación de espacios públicos y privados son temas relegados a un segundo plano, tanto por la representación política tradicional como por el activismo. De esta manera, muchas de las reivindicaciones con las que nacieron las agrupaciones sociales se han dejado en el olvido y ahora parece que los movimientos se conforman con los límites que les establece el orden político, siendo, en términos de Guerrero y Sutachán (2012), movimientos que se desplazan de la 
resistencia a la institucionalización a través de la consigna del reconocimiento de la diferencia.

El segundo punto adverso se refiere a cierto proceso de "conservadurismo" de la diversidad sexual. Al tiempo que se va consolidando una agenda de derechos, surge un nuevo orden moral que vigila la vida privada de sus destinatarios. El hecho de que el Estado tenga en sus manos la promoción política de la diversidad sexual le confiere la competencia de promover fronteras de legitimidad que demarcan las posibilidades de ser y de actuar dentro de ellas. Se crea, entonces, una especie de manual de convivencia que determina las formas de vida a las cuales se deben adscribir las sexualidades e identidades no normativas. De acuerdo a Boivin, "frente al modelo de vida de soltería y libertinaje, se introducen nuevos valores: la pareja estable, la fidelidad, la corresidencia y la conservación del patrimonio" (Boivin, 2011, p. 174). En este sentido, la institucionalización, o en otras palabras, la entrada a la democracia liberal, se olvida de muchas prácticas que hacen parte de la historia de la diversidad sexual, un olvido consciente que intenta borrar del mapa algunas prácticas que le resultan fatales a sus ojos. Asuntos como el cruising y los cuartos oscuros (prácticas entre varones que surgieron debido a la clandestinidad en la cual estuvo sumida la homosexualidad en el siglo pasado), la crítica a la monogamia heteroparental, las prácticas del leather y el BDSM (Bondage/Dominación/Sadomasoquismo), en suma, las prácticas "contra-sexuales" (Preciado, 2002), son temas que producen escozor al fundamento institucional de la diversidad sexual y pretenden ser anulados ${ }^{4}$.

Se evidencia, entonces, una comprensión sesgada de las sexualidades e identidades diversas, que no toma en cuenta algunos precedentes que aún tienen vigencia. Lo más preocupante es que la misma población integrada en la diversidad sexual fetichiza estos dictámenes creando jerarquías que señalan negativamente a las personas que no se acogen a la lógica de la institucionalización, siendo éste el tercer efecto perjudicial. Pareciera que actualmente se estuvieran reactualizando las posiciones dentro de la pirámide de estratificación sexual propuesta por Rubin (1999), que se determinan según la adscripción o no a los preceptos tipificados por la institucionalización. Quienes permanecen por fuera de este proceso -la "chusma sexual"- ya no sólo tienen que sobrellevar la discriminación por parte de la sociedad en general, sino que también tienen que soportar los señalamientos de los sectores LGBT:

Esta estigmatización recaería sobre la 'chusma sexual', que no se reconoce en esa agenda de ciudadanía: maricones promiscuos a los que no les interesa el

\footnotetext{
${ }^{4}$ Sobre el tema, Boivin señala que "en su voluntad de rehabilitar al homosexual, a partir de los años 1990 el discurso gay borra los elementos del pasado homosexual que ya no reconocía debido a que aquel ambiente marginal era desvalorizante" (Boivin, 2011, p. 176). De acuerdo con Gil Hernández (2013) esta estrategia moral tiene mucho sentido en la medida en que es necesario deconstruir un estereotipo estigmatizado que sustenta ideológicamente su discriminación y su falta de reconocimiento social e histórico
} 
matrimonio, travestis empobrecidas que ejercen la prostitución, machorras desempleadas que no pueden pagar impuestos, loquitas que no han podido estudiar, cuerpos queer que no son suficientemente blancos o burgueses (Gil Hernández, 2013, p. 64-65).

Esto además implica una adhesión a la heteronormatividad y cisnormatividad en la aceptación de la diversidad sexual y un debilitamiento de las posibilidades transgresoras de los actos performativos de género (Butler, 1998). Para que la diversidad sexual sea aceptada frente al "común de la población", es pertinente que se representen los roles tradicionales de género, así las identidades sexuales sean "diversas". De esta manera, usted puede ser una mujer lesbiana, pero debe conservar su feminidad, puede ser un hombre homosexual pero no puede actuar "afeminadamente"; es común en el argot gay de distintos países latinoamericanos escuchar los conceptos "serio", "varonil", "sin plumas", que operan como significantes de valor para posicionarse en determinado lugar privilegiado del deseo e incluso de aceptación y "éxito" social.

Por último, también es importante mencionar una cuarta situación que se ha generado a partir de estos silencios en la problematización de la diversidad sexual: la reciente apertura de las lógicas del mercado neoliberal contemporáneo a las poblaciones sexualmente diversas y la construcción de un llamado "mercado rosa" o gay-friendly. Como se ha demostrado a lo largo del artículo, el Estado-nación contemporáneo logra obtener más réditos, tanto políticos como económicos, administrando sus alteridades sexuales que, propendiendo por su erradicación, izando la lógica procedimental y praxiológica del capitalismo neocolonial: "sea mercancía y no muera en el intento" (Scribano, 2009).

La promoción de algunas ciudades y de ciertas zonas de ellas (“Zona Rosa” en México D.F., Chapinero en Bogotá, Bellas Artes/Lastarria en Santiago, por mencionar algunas) como espacios que ofrecen servicios especiales dirigidos a estas poblaciones, genera determinadas situaciones. Por un lado, se construyen imágenes publicitarias de las ciudades como "tolerantes" y "amigables" con la diversidad sexual, que son vendidas a nivel internacional para atraer turistas, pero que invisibilizan las condiciones de marginación y discriminación endógena que viven las poblaciones sexualmente diversas dentro de estas ciudades. Por otra parte, estas imágenes aluden a un "sujeto LGBT" específico: un sujeto varón con el capital económico y cultural para estar "a la moda" y con determinados intereses relacionados con el consumo de estéticas corporales (peluquerías, negocios de belleza corporal, gimnasios), de ocio y de diversión

\footnotetext{
${ }^{5}$ Esta "estratificación sexual" también aparece mediada, en el contexto latinoamericano que nos ocupa, por la imbricación mencionada anteriormente de categorías de discriminación, que articulan la diversidad sexual con la diversidad étnico-racial y cultural, así como con la condición de clase. De esta manera, y como lo señalan Viveros Vigoya (2009) y Gil Hernández (2008, 2013), las personas se construyen como sujetos de deseo (o de no deseo) caracterizadas por marcadores de género, raciales, culturales y de clase. Esta situación en las organizaciones de defensa de derechos étnico-raciales y culturales, es generalmente silenciada, ignorando las agendas de las minorías sexuales que las integran, y legitimando las estrategias de discriminación criticadas desde los análisis interseccionales.
} 
(restaurantes, bares, discotecas). ¿Qué pasa con las personas que no cumplen con estos cánones, que no tienen las condiciones materiales o simplemente no les interesa acceder al consumo de estos universos simbólicos? ¿No son parte de la "comunidad LGBT”? ¿No pueden integrarse a la ciudadanía formal?

\section{Consideraciones finales}

A partir de lo analizado a lo largo del texto, concluiremos por el momento que, de la misma forma que la diversidad sexual se manifiesta de distintas maneras según determinados contextos históricos y socioculturales, se debe reflexionar a través de nuevas y diferentes estrategias epistemológicas y metodológicas con el propósito de promover preceptos teóricos y políticos más amplios y de mayor pertinencia, que abarquen a las poblaciones con sexualidades e identidades diversas y que no se limiten a imágenes construidas desde la nominación del estado patriarcal heteronormativo. Las políticas promulgadas hasta ahora en la región no han incidido significativamente en una transformación del lugar de subordinación histórico de las poblaciones sexualmente diversas.

Sumado a lo anterior, la institucionalización filtra la diversidad sexual, haciendo de ella algo funcional a la lógica política de los Estados, desestimando muchas otras ideologías y prácticas suscritas históricamente a la diversidad sexual, por un lado, invisibilizándolas y olvidándolas, y por otro estableciendo una moral sexual para la diversidad como condición sine qua non para acceder a una ciudadanía formal dentro de estos Estados. Los sujetos sexualmente diversos debemos demostrar que reunimos las condiciones para poder ser ciudadanos(as) y acceder a los derechos y deberes propios de esta categoría, pero además de ello, debemos demostrar que somos personas "excepcionalmente ejemplares" para ser tratados como iguales: ser excelentes padres y madres (con una pareja establecida, en una relación monogámica, con condiciones socioeconómicas medias-altas) para poder formalizar procesos de adopción, ser excelentes consumidores de productos y servicios (es decir, tener trabajos estables, ingresos económicos medios-altos), ser excelentes ejemplos de conducta sexual y moral (evitar prácticas sexuales “desviadas"/estigmatizadas, mantener nuestras expresiones de afecto en privado), ser hombres o ser mujeres, pero no ubicarnos en un lugar de indefinición o ambigüedad amenazante. En suma, ser ciudadanos(as) ejemplares.

Finalmente, la centralidad que ha tomado el llamado "movimiento LGBT" en términos del activismo y la participación a través de demandas frente al Estado invisibiliza las prácticas y estrategias de la vida cotidiana que no están referidas a la participación en colectivos políticos que reivindican derechos de las personas sexualmente diversas (Aparicio Erazo, 2009). ¿Qué pasa con las personas que no están vinculadas a ninguna de estas organizaciones, pero que están demostrando su identidad 
sexual o de género abiertamente en la cotidianidad? ¿En qué lugar se ubican las personas que transgreden los roles heteronormativos y cisnormativos y que a la vez se encuentran en condiciones de discriminación por cuestiones étnicas, raciales o de clase? ¿Cómo están transformando las relaciones de dominación y exclusión? ¿Cómo operan las acciones afirmativas y estrategias de resistencia que realizan día a día? ¿Qué importancia tienen estas manifestaciones en las transformaciones de los imaginarios y representaciones del resto de la ciudadanía que presencian este tipo de prácticas en su cotidianidad? Estas son preguntas que, creemos, deben ser profundizadas y puestas en el lugar que se merecen en términos de la construcción de una ciudadanía sexual más incluyente, más justa y menos violenta frente a nuestras sexualidades e identidades diversas. 


\section{Referencias}

Anderson, B. (1993). Comunidades imaginadas. Ciudad de México: Fondo de Cultura Económica.

Aparicio Erazo, J. (2009). Ciudadanías y homosexualidades en Colombia. Quito: Iconos - Revista de Ciencias Sociales, 35, 43-54.

Boivin, R. (2011). De la ambigüedad del clóset a la cultura del gueto gay: género y homosexualidad en París, Madrid y México. Buenos Aires: La Ventana, 34, 146190.

Bustamante Tejada, W. (2013). Masculinidad y homofobia. El control de la sexualidad del varón en la construcción del Estado colombiano. Cali: Revista Sociedad y Economía, 24, 159-182.

Butler, J. (1998). Actos performativos y constitución del género: un ensayo sobre fenomenología y teoría feminista. Ciudad de México: Debate Feminista, 18, 296314.

De la Dehesa, R. (2010). Queering the public sphere in Mexico and Brazil. Sexual Rights Movements in emerging democracies. Durham: Duke University Press.

Díez, J. (2010). El movimiento lésbico-gay, 1978-2010. En: Tepichín, A., Tinat, K. y Gutiérrez, L. (Coords.). Los grandes problemas de México. Relaciones de Género. Ciudad de México: Colegio de México, 8, 135-154.

Dubel, I. y Hielkema, A. (2010). Urgency required: Gay and lesbian rights are human rights. Humanist Institute for Cooperation with Developing Countries (Hivos).

Estefan, S. (2013). Discriminación estatal de la población LGBT. Casos de transgresiones a los derechos humanos en Latino América. Cali: Revista Sociedad y Economía, 25, 183-204.

Fassin, E. (2009). Género, sexualidades y política democrática. Ciudad de México: UNAM-PUEG.

Gil Hernández, F. (2013). Fronteras morales y políticas sexuales: apuntes sobre 'la política LGBT' y el deseo del Estado. Río de Janeiro: Revista Sexualidad, Salud y Sociedad Latinoamericana, 13, 43-68.

Gil Hernández, F. (2008). Racismo, homofobia y sexismo. Reflexiones teóricas y políticas sobre interseccionalidad. En: Wade, P., Urrea Giraldo, F. y Viveros Vigoya, M. (2008). Raza, etnicidad y sexualidades. Ciudadanía y multiculturalismo en América Latina. Bogotá: Universidad Nacional de Colombia-CES. 
Guerrero, O. y Sutachán, H. (2012). "En Colombia se puede ser...": indagaciones sobre la producción de lo LGBT desde la academia. Bogotá: Nómadas, 37, 219-229.

Hill Collins, P. (2004). Black sexual politics. African Americans, gender, and the new racism. Londres: Routledge.

Maceira, A. y Rayas, L. (2007). El entorno internacional y la IPEG. En: Ochoa, L., Alva, B. y Rayas, L. (2007). Elementos para el análisis de los procesos de institucionalización de la perspectiva de género: una guía. Ciudad de México: Colegio de México-Programa Interdisciplinario de Estudios de la Mujer.

MacKinnon, C. (1995). Hacia una teoría feminista del Estado. Madrid: Editorial Cátedra. Mouffe, C. (1999). El Retorno de lo Político. Barcelona: Paidós.

Nagel, J. (2000). Ethnicity and sexuality. Annual Review of Sociology, 26, 107- 133.

Núñez Noriega, G. (2011). ¿Qué es la diversidad sexual? Reflexiones desde la academia y el movimiento ciudadano. Quito: Ediciones Abya-Yala.

Pateman, C. (1996). Criticas feministas a la dicotomía público/privado. En: Castells, C. (Coord.). Perspectivas feministas en teoría política. Barcelona: Paidós.

Pateman, C. (1995). El contrato sexual. Barcelona: Anthropos.

Phillips, A. (1996). ¿Deben las feministas abandonar la democracia liberal?. En: Castells, C. (Coord.). Perspectivas feministas en teoría política. Barcelona: Paidós.

Preciado, B. (2002). Manifiesto contra-sexual. Madrid: Ópera Prima.

Rubin, G. (1999). Reflexionando sobre el sexo: notas para una teoría radical de la sexualidad. En: Vance, C. (Comp.). Placer y peligro: explorando la sexualidad femenina. Madrid: Talasa Ediciones.

Scribano, A. (2009). ¿Por qué una mirada sociológica de los cuerpos y las emociones? En: Scribano, A. y Figari, C. (Comps.) (2009). Cuerpo(s), subjetividad(es) y conflicto(s). Hacia una sociología de los cuerpos y las emociones desde Latinoamérica. Buenos Aires: CLACSO-CICCUS.

Vieira, J. y Pires, I. (2014). Políticas públicas e crítica queer: algumas questões sobre identidades LGBT. Belo Horizonte: Psicologia \& Sociedade, 2(26), 290-300.

Viveros Vigoya, M. (2009). La sexualización de la raza y la racialización de la sexualidad en América Latina. Manizales: Revista Latinoamericana de Estudios de Familia, $1,63-81$.

Wade, P., Urrea Giraldo, F. y Viveros Vigoya, M. (2008). Raza, etnicidad y sexualidades. Ciudadanía y multiculturalismo en América Latina. Bogotá: Universidad Nacional de Colombia-CES.

Wade, P. (2013). Racismo, democracia racial, mestizaje y relaciones sexo/género. Bogotá: Tábula Rasa, 18, 45-74. 